\title{
The Medieval Imaginary, a mirror of our thoughts
}

\section{Luisa Ţuculeanu}

Laura Mesina, Imaginarul medieval, Forme si teorii / The Medieval Imaginary, Editura Institutului European, Iași, 2015.

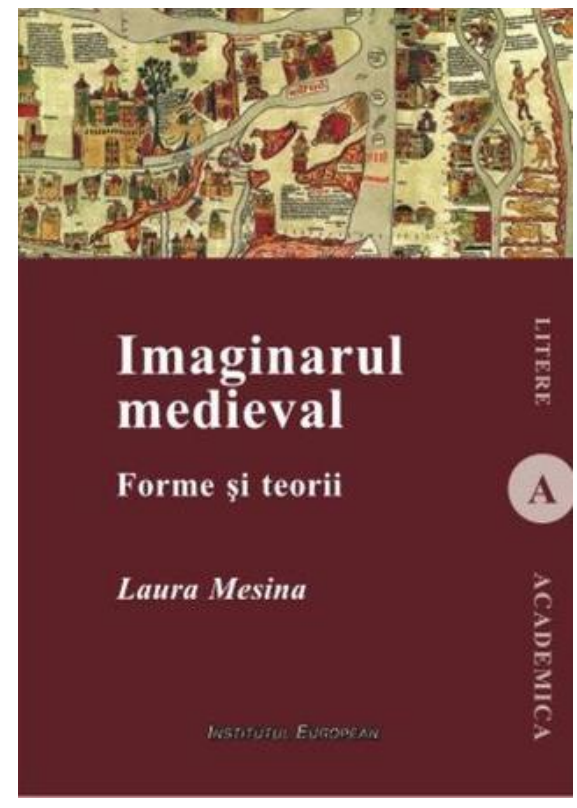

The Medieval Imaginary offers a theoretical introduction to the Middle Ages. From the very beginning one can notice the author's prime concern with the meaning of the Latin word "speculum" (equivalent to the noun "mirror"), which just shows that "speculum" is seen as a keyword for the entire study, having the role of a veritable guiding concept. The ordinary sense of this significant word is a jumping-off point for the author, who takes into account the idea of rendering and reproducing exterior details through images.

The author of this book, Laura Mesina, points out that the medieval universe offers a specific stability to its collective imaginary, due to a certain constancy of some reference points, such as duration, spiritual and religious life (including sacred figures, images, symbols etc.). In a broad sense, the concept has been studied by Descartes and later on by S. Freud and his disciple, C.G. Jung, but Gilbert Durand was the one who integrated this notion (otherwise, hard to define) into the area of the scientific vocabulary in the 1960's. Consequently, the starting point of this research is to identify above all the aspects of a theoretical nature. "The Theory", says the author, refers to the contemplation of ideas and that of the imaginary itself. In addition, it is likely to give various and comprehensive interpretations to this phenomenon. That is why, the author aims at giving us a new perspective, a different way of "reading" the medieval imaginary that can sharpen our understanding, by resorting to the metaphor of the "mirror". In fact, Speculum Speculorum, the first chapter of this book, makes reference to this 
symbol. The image of a mirror is taken over from another mirror, which suggests the distance between the interpretation regarding the imaginary and some familiar theories. The imaginary may be seen as a narrative way for other spheres of activity, like history and politics, as well. Moreover, there can be drawn a comparison between this concept and the system of language, the latter being viewed as a manner of "translation" concerning one's representations. In this context, archetypes, having a major role within the economy of the imaginary, are being analyzed from the words' perspective, particularly their form, which is similar to a certain extent. The purpose of this book is not, however, to emphasize the imaginary as a literary phenomenon, but to highlight the idea of the collective imaginary, rendered evident by the discourse of the authority, in a political manner.

The Orthodox imaginary (analyzed in the third chapter, Speculum mundi) is beyond doubt in the service of religion. Besides, the Christianity emerges as a spiritual solution in order to cope with social forces. The Byzantine imaginary is an example of great value for our medieval/Orthodox imaginary. Naturally, this aspect is adapted to the collective background, as well as to the Wallachian culture. The reference is to that period of the fourteenth century (Transylvania) which continues for Wallachia until the Revolution in 1821. In other words, the government forms created in the fourteenth century substantially influenced the Orthodox belief, including the developing authority according to an ecclesiastic plan. Thus, the perfect example for the first Christian emperor corresponds to this very plan (Neagoe Basarab is often thought of as being the major Orthodox model).

The Chamber of the Mirrors, the title of the fourth chapter, is a metaphor used to put together each and every reflected image. One thing to be remarked is that the medieval imaginary hasn't changed much in comparison with the Romanian society, for instance. When it comes to the Western countries, the medieval context is different, especially in scenography. That is to say it is more dynamic in the modern context. On top of that, the relationship between the individual and the divinity is strongly recognized. On the other hand, there is the doctrine of the iconoclasts which refuses the existence of a connection between the sacred personage and his corresponding image. Furthermore, in the sixteenth century the debates were still centered on the importance of the image. However, concludes the author, the faith symbolizes the collective thinking in general.

Needless to say, the medieval imaginary is a very generous topic and quite difficult to define. Therefore, Jacques Le Goff's study is relevant from this perspective, offering at the same time some important theoretical reference points. We should mention that the present study greatly influenced Laura Mesina in her analysis on Middle Ages, even if she outstrips his conception at one time. For instance, the medievalist suggests that representations are a sort of interface between reality and imaginary, being 
created by the help of the mimetic imagination, symbolic images and fantasy. Among other things, Le Goff agrees with the idea that the written text changes its status, so that the text is twofold: firstly, it is regarded as evidence, secondly, it indicates the sense of discourse. In short, they both represent significances of the past, as well as meanings of the future. Thus, the medieval imaginary (within the Wallachian background) is seen as a system which enables the community to build its own identity. Accordingly, that is why writings about the community's past are principally analyzed, as a keen reflection of both individual and collective conscience.

As for the modern theories dealing with the imaginary, the collective memory doesn't stand no longer for a witness of a continuous history, but it's incoherent and diverse, too. As a matter of fact, time itself is fragmented. However, there are some constant aspects of the Middle Ages with respect to the collective mentality. When it comes to Wallachian Middle Ages, the author refers to a long period of time, which ranges from the fourteenth century to the eighteenth century. This period of time is characterized by political ideologies and representations of the power that do not change significantly over the years. Representations of the collective mentality are defined by the imagination, divided into three parts, as follows: the mimetic imagination, the symbolic imagination (which is concerned with the sacred part acting dogmatically) and the fantasy (as to the Wallachian Middle Ages, the folk Christian imaginary is actually relevant, as well as some important literary writings).

All in all, the subject matter of the writer is to define the medieval imaginary as a whole, in spite of all particular, frequently changing contexts. Nevertheless, the author's conclusion is that beyond some fixed structures, an universal form cannot exist but in theory. However, the collective imaginary has enriched over the years, but most of all, has continued its history. Finally, Laura Mesina seems to ask herself whether the imaginary's controversial aspects emerge or not from our anxiety and fear of the unknown. 